\title{
Pied-Piping in Relative Clauses: Syntax and Compositional Semantics based on Synchronous Tree Adjoining Grammar
}

\author{
Chung-hye Han \\ Department of Linguistics \\ Simon Fraser University \\ chunghyedsfu.ca
}

\begin{abstract}
In relative clauses, the $w h$ relative pronoun can be embedded in a larger phrase, as in a boy [whose brother] Mary hit. In such examples, we say that the larger phrase has pied-piped along with the whword. In this paper, using a similar syntactic analysis for $w h$ pied-piping as in Han (2002) and further developed in Kallmeyer and Scheffler (2004), I propose a compositional semantics for relative clauses based on Synchronous Tree Adjoining Grammar. It will be shown that (i) the elementary tree representing the logical form of a $w h$-word provides a generalized quantifier, and (ii) the semantic composition of the pied-piped material and the $w h$-word is achieved through adjoining in the semantics of the former onto the latter.
\end{abstract}

\section{Introduction}

In relative clauses, the $w h$ relative pronoun can be embedded in a larger phrase, as in (1) and (2). In such examples, we say that the larger phrase containing the $w h$-word has PIED-PIPED along with the $w h$-word.

(1) a boy $[\text { [whose brother }]_{i}$ Mary hit $\left.\mathrm{t}_{i}\right]$

(2) a boy [[whose brother's friend $]_{i}$ Mary hit $\left.t_{i}\right]$

In this paper, using a similar syntactic analysis for $w h$ pied-piping as in Han (2002) and further developed in Kallmeyer and Scheffler (2004), I propose a compositional semantics for relative clauses of the sort illustrated in (1) and (2), based on Synchronous Tree Adjoining Grammar (STAG). The two main components of my proposal are that (i) the semantic tree representing the logical form of a $w h$ relative pronoun provides a generalized quantifier, and (ii) the semantic composition of the pied-piped material and the $w h$-word is achieved through adjoining of the former onto the latter in the semantics. Although TAG semantics for relative clauses based on flat semantics have been proposed before (Han, 2002; Kallmeyer, 2003), no STAG-based analysis exists, as far as I know.

In section 2, I introduce the framework of STAG and STAG-based compositional semantics and clarify my assumptions. In section 3, I present my analysis of relative clauses and pied-piping. I extend the proposed analysis to relative clauses in which $w h$-word is in a PP and those in which no pied-piping has taken place in section 4 .

\section{STAG-based Compositional Semantics}

Before presenting my analysis of relative clauses, I first illustrate the framework of STAG-based compositional semantics and clarify my assumptions, using a simple sentence that contains an existential quantifier and an attributive adjective in (3).

(3) John saw a good movie.

I use STAG as defined in Shieber (1994). In an STAG, each syntactic elementary tree is paired with one or more semantic trees that represent its logical form with links between matching nodes. A synchronous derivation proceeds by mapping a derivation tree from the syntax side to an isomorphic derivation tree in the semantics side, and is synchronized by the links specified in the elementary tree pairs. In the tree pairs given in Figure 1, the trees in the left side are syntactic elementary trees and the ones in the right side are semantic trees. In the semantic trees, F stands for formulas, $\mathrm{R}$ for predicates and $\mathrm{T}$ for terms. I assume that these nodes are typed and I represent predicates as unreduced $\lambda$-expressions. The linked nodes are shown with boxed numbers. For sake of simplicity, in the elementary tree pairs, I only include links that are relevant for the derivation of given examples.

Figure 1 contains elementary trees required to generate the syntactic structure and the logical 


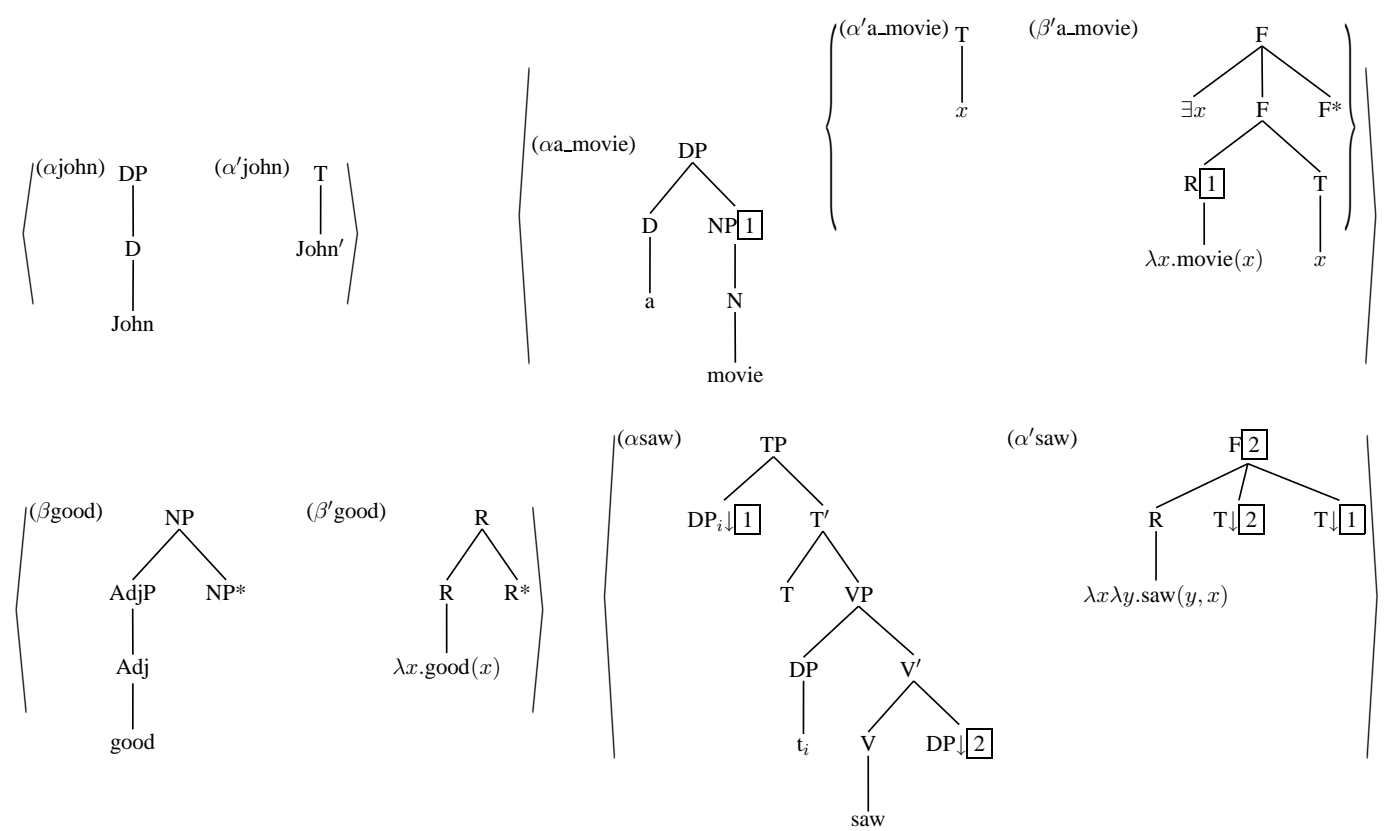

Figure 1: Elementary trees for John saw a good movie.

form of (3). All the syntactic elementary trees satisfy Frank's (2002) Condition on Elementary Tree Minimality (CETM), which states that "the syntactic heads in an elementary tree and their projections must form an extended projection of a single lexical head" (Frank 2002, p. 54). Particularly, ( $\alpha$ a_movie) is a valid elementary tree, as a noun can form an extended projection with a DP, in line with the DP Hypothesis. The proper name tree in $(\alpha \mathrm{John})$ is paired with a tree representing a term in the semantics, and the attributive adjective tree in $(\beta$ good) is paired with an auxiliary tree in the semantics that represents a one-place predicate to be adjoined to another one-place predicate. As for the syntax-semantics pairing of elementary trees for quantified DPs, I follow Shieber and Schabes (1990), and use Tree Local Multi-Component TAG (as defined in Weir (1988)) in the semantics. Thus, the DP in ( $\alpha$ a_movie) is paired with a multi-component set $\left\{\left(\alpha^{\prime}\right.\right.$ a_movie $),\left(\beta^{\prime}\right.$ a_movie $\left.)\right\}$ in the semantics: ( $\alpha^{\prime}$ a_movie) provides an argument variable, and ( $\beta^{\prime}$ a_movie) provides the existential quantifier with the restriction and scope. The transitive tree in ( $\alpha$ saw) is paired with a semantic tree representing a formula that consists of a two-place predicate and two term nodes. The links, shown with boxed numbers, guarantee that whatever substitutes into $\mathrm{DP}_{i}$, the corresponding semantic tree will substitute into the term node marked with 1 , and whatever substitutes into DP is paired up with a multi-component set in the se- mantics where one of the components will substitute into the term node marked with 2 and the other will adjoin onto the $\mathrm{F}$ node marked with 2 . The syntactic and semantic derivation trees are given in Figure 2, and the derived trees are given in Figure 3. I leave out the tree addresses in the semantic derivation tree, as these are determined by the links between the syntactic and semantic elementary trees. ${ }^{1}$
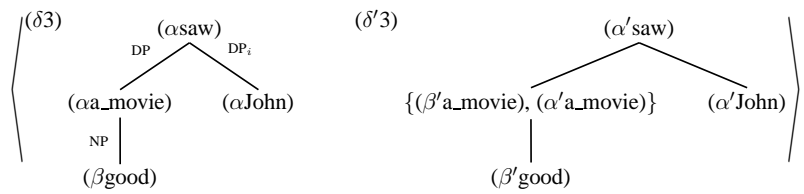

Figure 2: Derivation trees for John saw a good movie.

The semantic derived trees can be reduced by applying $\lambda$-conversion, as the nodes dominate typed $\lambda$-expressions and terms. When reducing semantic derived trees, in addition to $\lambda$-conversion, I propose to use Predicate Modification, as defined in Heim and Kratzer (1998) in (4).

(4) Predicate Modification

If $\alpha$ has the form

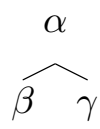

\footnotetext{
${ }^{1}$ In sentences with more than one quantified DPs, I assume multiple adjoining (as defined in Schabes and Shieber (1994)) of quantifier trees at the same F node, leaving the order unspecified. This provides an underspecified representation and accounts for scope ambiguity.
} 


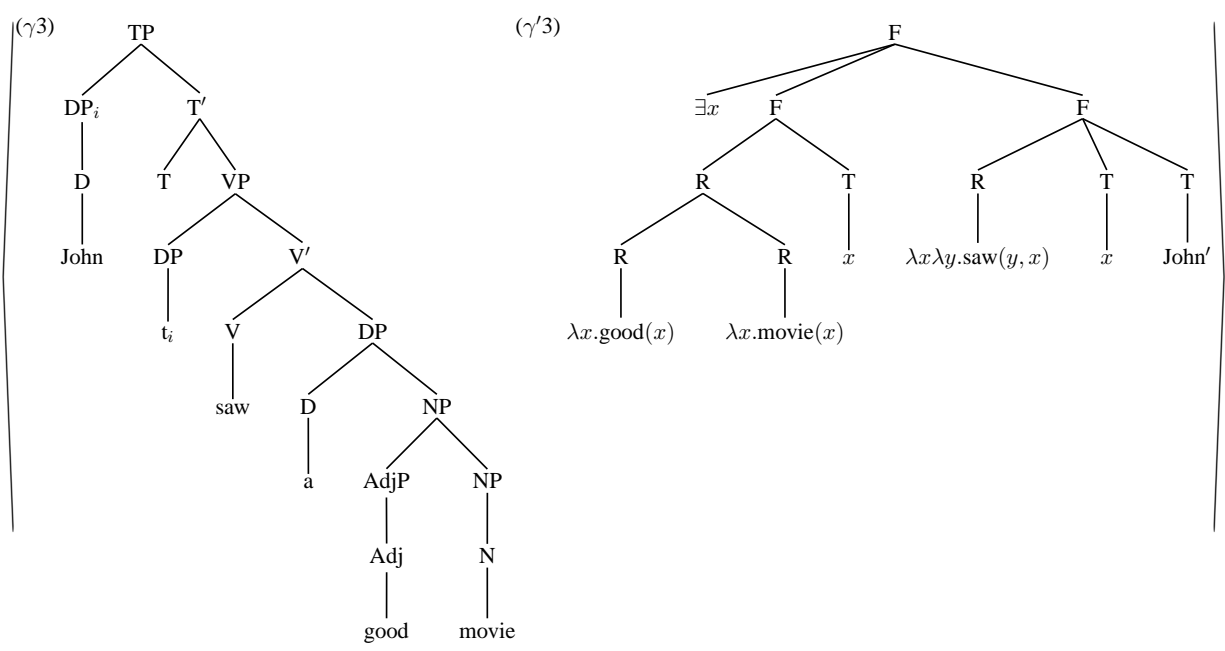

Figure 3: Derived trees for John saw a good movie.

and $\llbracket \beta \rrbracket^{s}$ and $\llbracket \gamma \rrbracket^{s}$ are both in $D_{<e, t>}$, then $\llbracket \alpha \rrbracket^{s}=\lambda x_{e} \llbracket \beta \rrbracket^{s}(x) \wedge \llbracket \gamma \rrbracket^{s}(x)$.

The application of Predicate Modification and $\lambda$ conversion to $\left(\gamma^{\prime} 3\right)$ reduces it to the formula in (5).

(5) $\exists x[\operatorname{good}(x) \wedge \operatorname{movie}(x)]\left[\operatorname{saw}\left(\operatorname{John}^{\prime}, x\right)\right]$

\section{An STAG analysis of pied-piping in relative clauses}

I propose the elementary tree pairs in Figure 4 for the syntactic derivation and semantic composition of the relative clause in (1). In the syntax side, ( $\alpha$ who) substitutes into $\mathrm{DP}_{j}$ in ( $\beta$ hit), and the pied-piping of the rest of the DP is achieved by adjoining ( $\beta$ 's brother) onto ( $\alpha$ who). The tree in ( $\beta$ 's brother) is a widely-accepted genitive structure according to the DP hypothesis, where the genitive 's heads the DP tree. This satisfies CETM, as a DP is an extended projection of a noun. Substituting ( $\alpha$ mary) into $\mathrm{DP}_{i}$ in ( $\beta$ hit) completes the derivation of the relative clause.

The derivation tree for the relative clause is in $(\delta 1)$ in Figure 5 and the derived tree is in $(\gamma 1)$ in Figure 6.
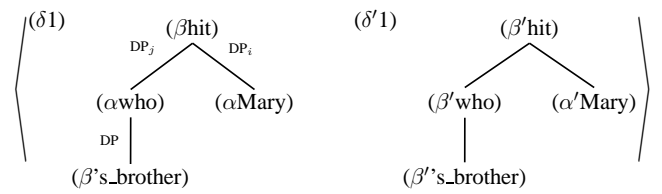

Figure 5: Derivation trees for whose brother Mary hit

Semantically, we must make sure that the variable coming from the $w h$-word is also the one being predicated of the head noun (boy in (1)), and yet the same variable does not serve as an argument of the predicate (hit in (1)) in the relative clause. I argue that the introduction of a generalized quantifier (GQ) node in the semantic tree in ( $\beta^{\prime}$ who) and adjoining of ( $\beta^{\prime}$ 's_brother) onto the GQ node guarantee this. I define the logical form of a $w h$ relative pronoun as an auxiliary tree given in ( $\beta^{\prime}$ who). In ( $\beta^{\prime}$ who $), \lambda x$ binds $x$ in the generalized quantifier, $\lambda P . P(x)$. Adjoining ( $\beta^{\prime}$ who) onto the relative clause elementary tree in $\left(\beta^{\prime}\right.$ hit) essentially has the effect of abstracting over the variable coming from the $w h$-word in the relative clause, turning it into a one-place predicate. This therefore ensures that the relative clause and the head noun are predicating over the same variable, deriving the interpretation of the relative clause as a modifier of the head noun. The meaning of the pied-piped material 's brother is added onto the meaning of who by adjoining the auxiliary tree defined in ( $\beta^{\prime}$ 's_brother) onto the GQ node in ( $\beta^{\prime}$ who). In ( $\beta^{\prime}$ 's_brother), $\lambda y$ ensures that the variable coming from the DP* (who) is in some relation with the variable coming from the head of the pied-piped DP (whose brother), and $\lambda Q$, by turning whose brother into a GQ, ensures that the variable coming from the head of the pied-piped DP is the argument of the predicate that the DP combines with. The derivation tree and the derived tree in the semantics side are given in $\left(\delta^{\prime} 1\right)$ in Figure 5 and $\left(\gamma^{\prime} 1\right)$ in Figure 6. After all the $\lambda$ conversions have applied, $\left(\gamma^{\prime} 1\right)$ can be reduced to the expression in (6).

(6) $\lambda x \cdot \operatorname{THE} z_{1}\left[\operatorname{brother}\left(z_{1}\right) \wedge\right.$ $\left.\operatorname{Rel}\left(x, z_{1}\right)\right]\left[\operatorname{hit}\left(\operatorname{Mary}^{\prime}, z_{1}\right)\right]$ 


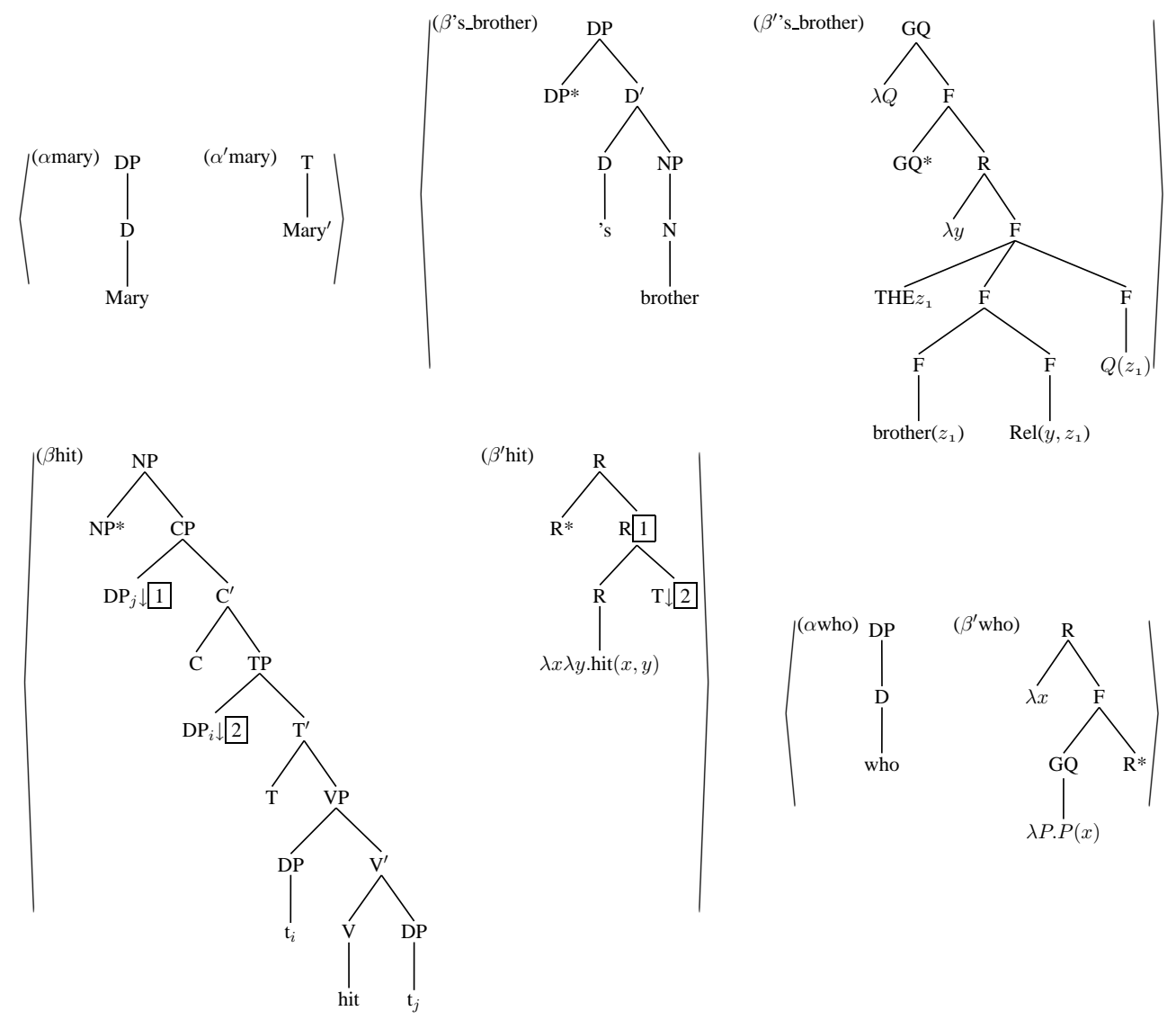

Figure 4: Elementary trees for whose brother Mary hit
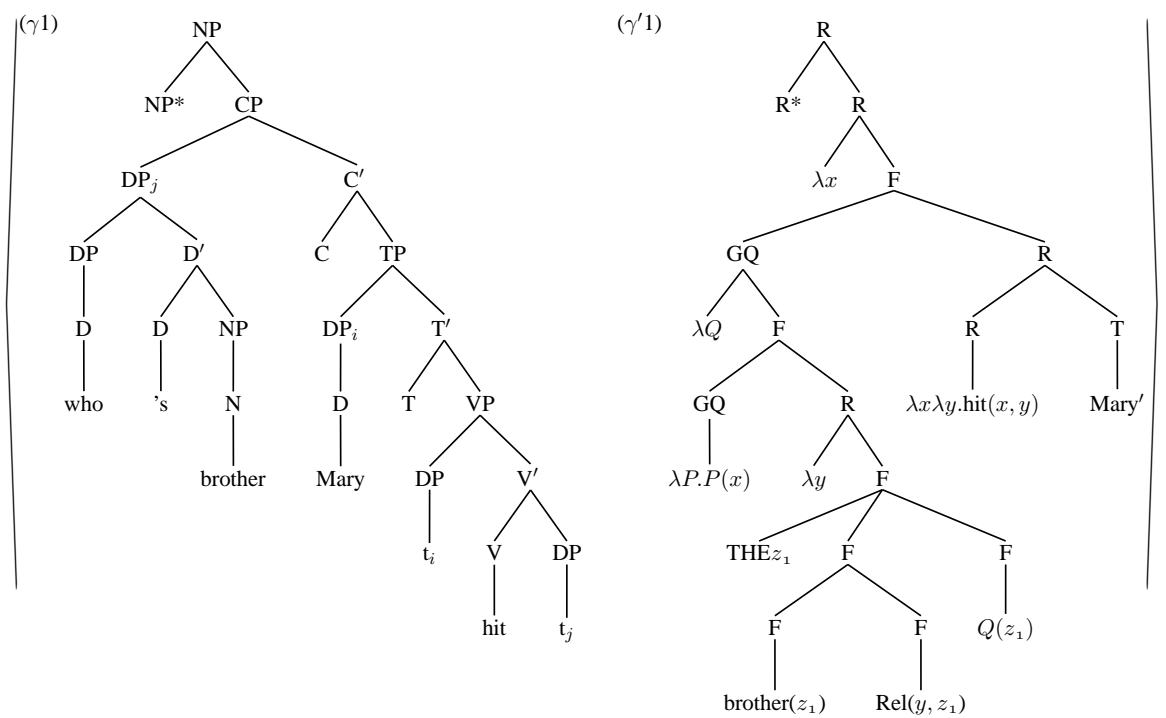

Figure 6: Derived trees for whose brother Mary hit 
The expression in (6) is a one-place predicate which can be paraphrased as a set of all $x$ 's such that there is a unique brother $z_{1}$ and $x$ is in some relation with $z_{1}$ and Mary hit $z_{1}$. As the semantics of relative clauses is defined to be a one-place predicate, it is analogous to attributive adjectives. This means that the semantic tree resulting from the adjoining of $\left(\gamma^{\prime} 1\right)$ onto the logical form of the head noun boy can be reduced to the expression in (7) through Predication Modification.

(7) $\lambda x \cdot \operatorname{boy}(x) \wedge \mathrm{THE} z_{1}\left[\operatorname{brother}\left(z_{1}\right) \wedge\right.$ $\left.\operatorname{Rel}\left(x, z_{1}\right)\right]\left[\operatorname{hit}\left(\operatorname{Mary}^{\prime}, z_{1}\right)\right]$

The derivation of a sentence containing (1), $a$ boy whose brother Mary hit, as the object, as in (8), proceeds in a similar fashion as in (3), yielding the semantic derived tree which is reducible to the formula in (9).

(8) John saw a boy whose brother Mary hit.

(9) $\exists x\left[\operatorname{boy}(x) \wedge \mathrm{THE} z_{1}\left[\operatorname{brother}\left(z_{1}\right) \wedge\right.\right.$ $\left.\left.\operatorname{Rel}\left(x, z_{1}\right)\right]\left[\operatorname{hit}\left(\operatorname{Mary}^{\prime}, z_{1}\right)\right]\right]\left[\operatorname{saw}\left(\operatorname{John}^{\prime}, x\right)\right]$

For the syntactic derivation and the compositional semantics of the relative clause in (2), all we need to do is add the tree pair in Figure 7 to the set of elementary tree pairs in Figure 4. In the syntax side, ( $\beta$ 's_friend) adjoins onto ( $\beta$ 's_brother) and in the semantics side, ( $\beta^{\prime}$ 's_friend) adjoins onto ( $\beta^{\prime}$ 's_brother), as shown in the derivation trees in Figure 8. The derived trees are given in Figure 9. The semantic derived tree $\left(\gamma^{\prime} 2\right)$ can be reduced to the expression in (10) through $\lambda$-conversions.

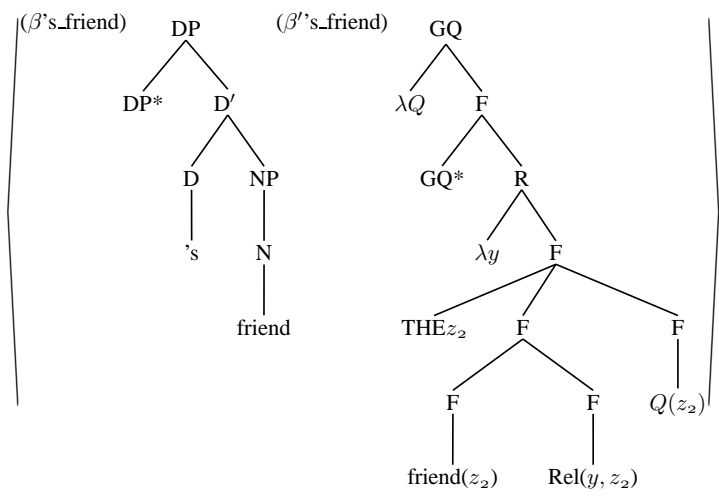

Figure 7: Elementary trees for 's friend

(10) $\lambda x \cdot \operatorname{THE} z_{1}\left[\operatorname{brother}\left(z_{1}\right) \wedge\right.$

$\left.\operatorname{Rel}\left(x, z_{1}\right)\right]\left[\operatorname{THE} z_{2}\left[\operatorname{friend}\left(z_{2}\right) \wedge\right.\right.$

$\left.\left.\operatorname{Rel}\left(z_{1}, z_{2}\right)\right]\left[\operatorname{hit}\left(\operatorname{Mary}^{\prime}, z_{2}\right)\right]\right]$
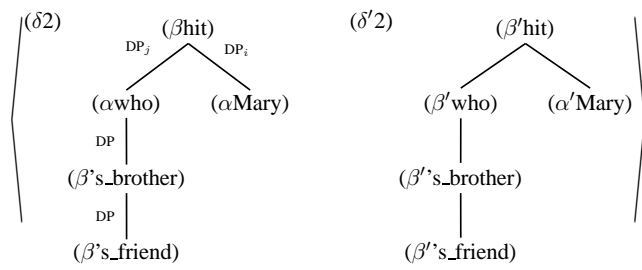

Figure 8: Derivation trees for whose brother's friend Mary hit

\section{Extensions}

The proposed syntax and the semantics of piedpiping can straightforwardly be extended to cases in which the $w h$-word is embedded in a PP, as in (11).

(11) a boy $\left[{ }_{D P} \text { the brother of whom }\right]_{i}$ Mary hit $\left.\mathrm{t}_{i}\right]$

For the derivation of (11), we need to change two of the elementary tree pairs in Figure 4 slightly. The elementary tree pairs $<(\alpha$ who $),\left(\beta^{\prime}\right.$ who $)>$ and $<(\beta$ 's_brother $), \beta^{\prime}$ 's_brother $)>$ need to be replaced with the pairs in Figure 10. Since the relative pronoun in (11) is whom, we use a DP tree anchoring whom in ( $\alpha$ whom). The corresponding semantic tree ( $\beta^{\prime}$ whom) remains exactly the same as before. ( $\beta$ the_brother_of) represents the piedpiped material in DP. It is a well-formed elementary tree according to CETM as it has a single lexical head brother and DP is an extended projection of this head, and PP is not subject to CETM because $\mathrm{P}$ is a functional head, not a lexical head. Moreover, DP* is licensed as it is an argument of the lexical head brother, as argued in Kroch (1989). The semantics of the brother of whom is equivalent to whose brother, and therefore, we pair up ( $\beta$ the_brother_of) with the exact same semantic tree as ( $\beta^{\prime}$ 's_brother).

The derivation trees for the relative clause in (11) are given in Figure 11. They look exactly the same as the ones for the relative clause in (1), except for names of the elementary trees in a few nodes. The derived trees are given in Figure 12. While the syntactic derived tree $(\gamma 11)$ is different from $(\gamma 1)$ in Figure 6 in the structure of DP containing the pied-piped material, the semantic derived tree $\left(\gamma^{\prime} 11\right)$ looks exactly the same as $\left(\gamma^{\prime} 1\right)$ in Figure 6. This is as it should be given that the meaning of (1) and the meaning of (11) are equivalent. 

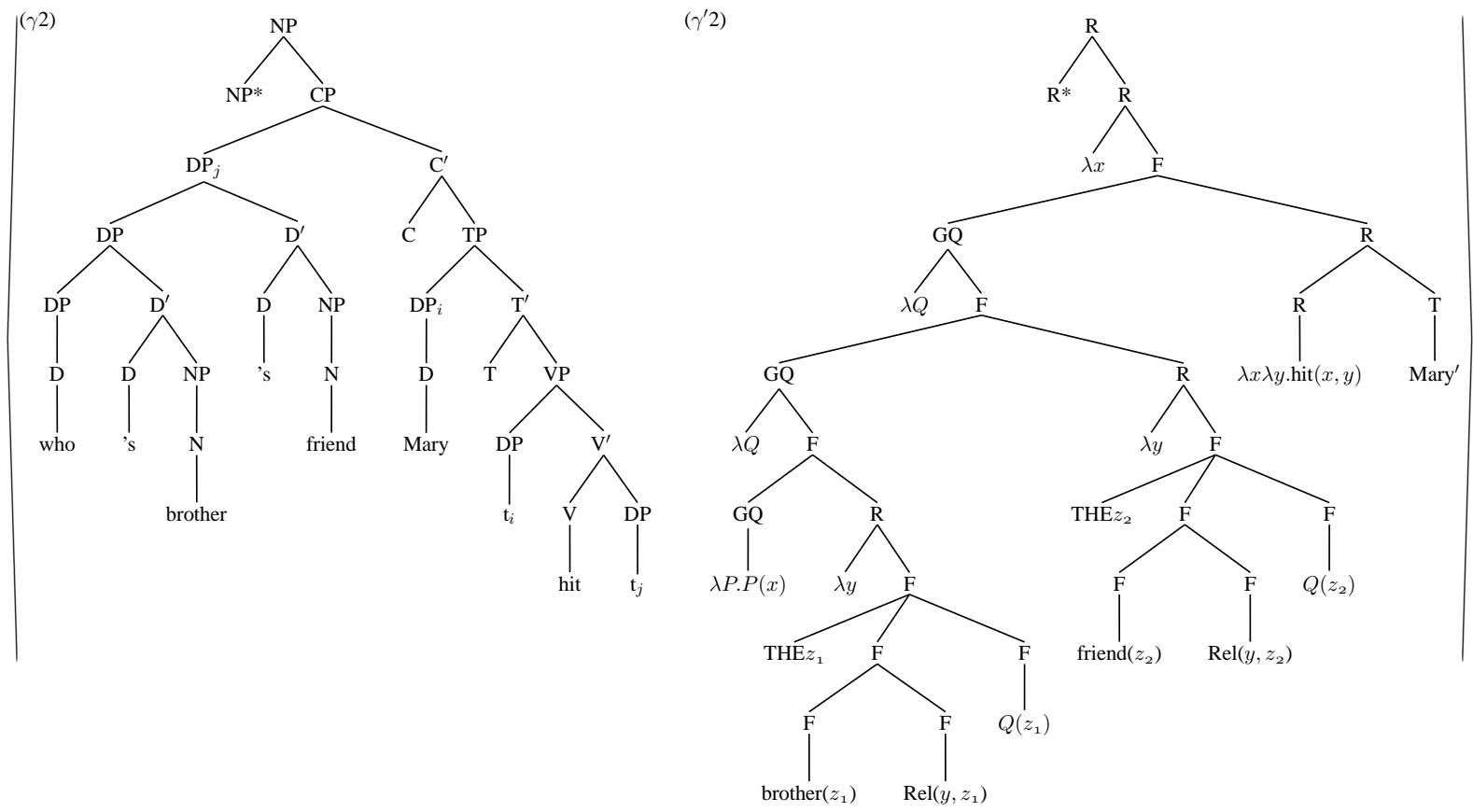

Figure 9: Derived trees for whose brother's friend Mary hit
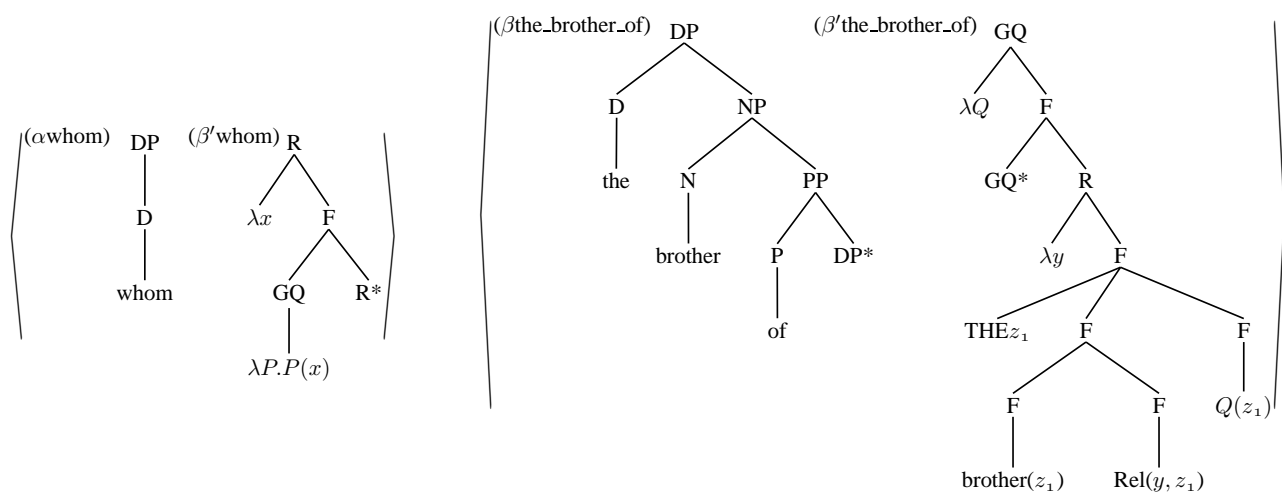

Figure 10: Elementary trees for whom and the brother of
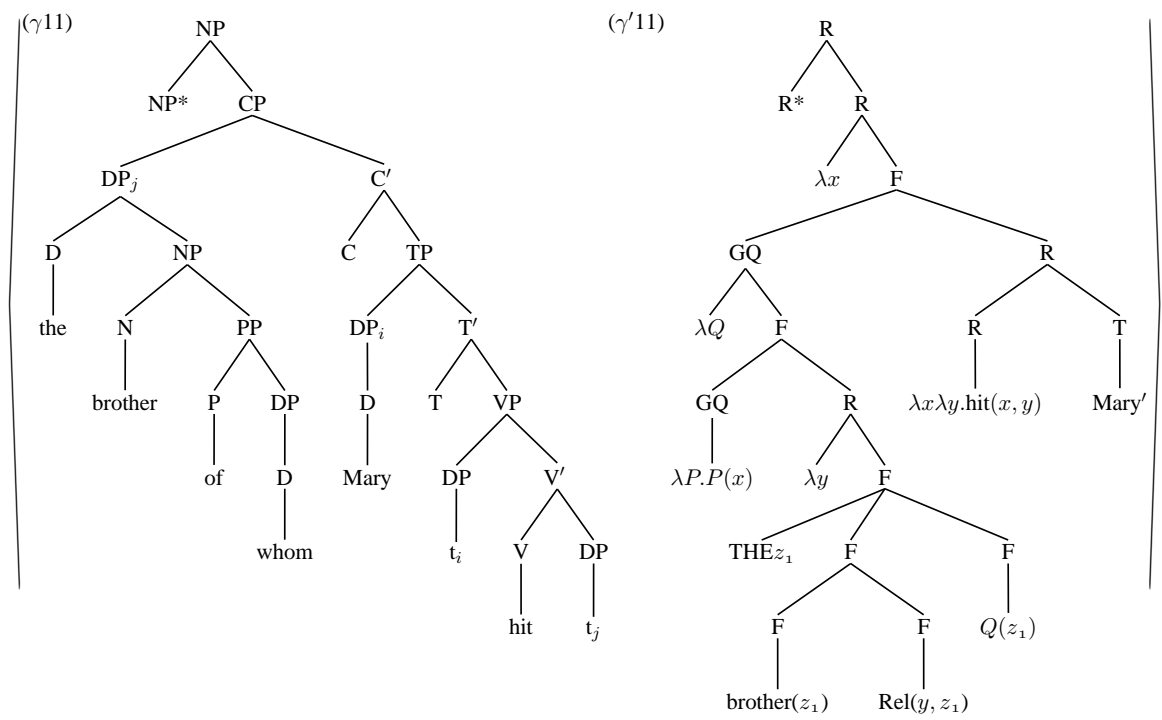

Figure 12: Derived trees for the brother of whom Mary hit 

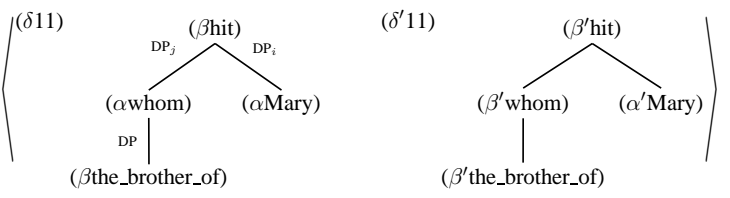

Figure 11: Derivation trees for the brother of whom Mary hit

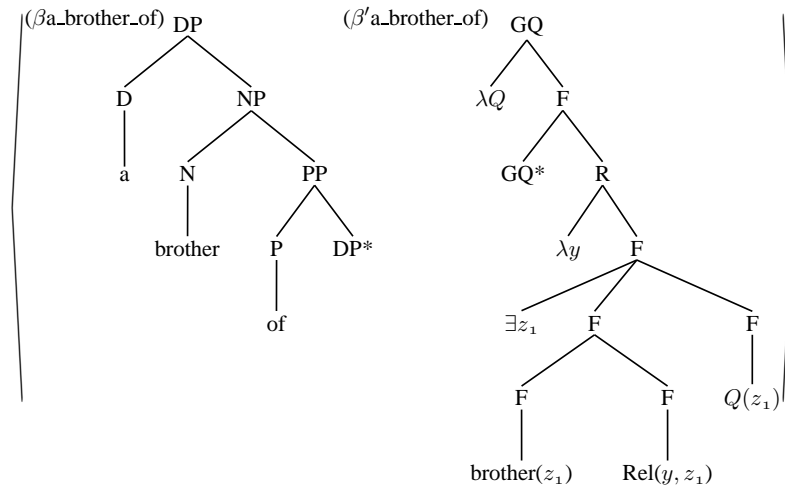

Figure 13: Elementary trees for whom and $a$ brother of

The proposed analysis can also be extended to relative clauses in which no pied-piping has taken place. When the larger DP containing the relative pronoun is indefinite or non-specific, the DP can be stranded, as in (12). This gives us a configuration where a $w h$-word has extracted out of a DP.

(12) a boy $\left[w_{h o m}\right.$ Mary hit [DP a brother of $\left.\left.t_{i}\right]\right]$

Since we now have a DP with an indefinite article, a tree pair in Figure 13 is needed, for the derivation of (12). Using the semantic tree ( $\beta^{\prime}$ a_brother_of), the semantic composition of the relative clause in (12) can proceed as before: the semantic tree ( $\beta^{\prime}$ a_brother_of) adjoins onto the semantic tree $\left(\beta^{\prime}\right.$ whom) in Figure 10, which then adjoins onto ( $\beta^{\prime}$ hit) in Figure 4. In the syntax, however, we must make sure that ( $\beta$ a_brother_of) does not adjoin onto the relative pronoun whom, because if it did, we would end up with the string a brother of whom. Instead, what we need is for ( $\beta$ a_brother_of) to adjoin onto the DP dominating the trace of the extracted object in ( $\beta$ hit). This however is not a valid derivation in STAG, as elementary trees in a single pair are composing with two trees from two different pairs. A slight modification in the syntactic elementary tree for ( $\alpha$ whom) in Figure 14 can fix this problem. I propose to do this by turning ( $\alpha$ whom) into a multicomponent set $\{(\alpha$ whom $),(\beta$ whom $)\}$ as in Figure 14. An auxiliary tree like ( $\beta$ whom), which

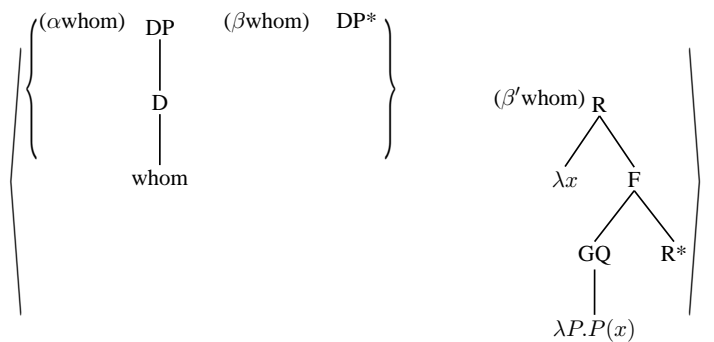

Figure 14: Elementary trees for whom
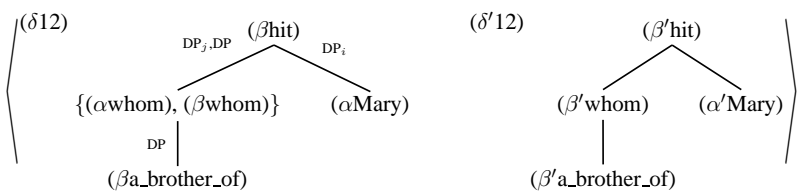

Figure 15: Derivation trees for whom Mary hit a brother of

does not dominate any other nodes, is a degenerate tree, and has been used in Kroch (1989) and Frank (2002) to handle extraction from a $w h$-island, as in [Which car] $]_{i}$ does Sally wonder how to fix $t_{i}$ ?

In syntax, to derive the relative clause in (12), ( $\alpha$ whom) substitutes into $\mathrm{DP}_{j}$ in $(\beta$ hit) as before, and ( $\beta$ whom) adjoins onto the DP dominating the trace of the extracted object in ( $\beta$ hit), as shown in the derivation tree $(\delta 12)$ in Figure 15. And in semantics, ( $\beta^{\prime}$ whom $)$ adjoins onto $\left(\beta^{\prime}\right.$ hit) as before, as shown in $\left(\delta^{\prime} 12\right)$ in Figure 15 . Subsequently, in syntax ( $\beta$ a_brother_of) adjoins onto ( $\beta$ whom) giving us the DP a brother of $t_{j}$, and in semantics ( $\beta^{\prime}$ a_brother_of) adjoins onto ( $\beta^{\prime}$ whom). Thus, by using the multi-component set $\{(\alpha$ whom $),(\beta$ whom $)\}$, we now have a situation where two elementary trees in a single pair are composing with two trees belonging to another pair. The syntactic and the semantic derived trees are given in Figure 16. After $\lambda$-conversions, $\left(\gamma^{\prime} 12\right)$ can be reduced to the expression in (13). ${ }^{2}$

$$
\begin{aligned}
& \lambda x . \exists z_{1}\left[\operatorname{brother}\left(z_{1}\right) \wedge\right. \\
& \left.\operatorname{Rel}\left(x, z_{1}\right)\right]\left[\operatorname{hit}\left(\operatorname{Mary}^{\prime}, z_{1}\right)\right]
\end{aligned}
$$

\section{Conclusion}

I have shown that STAG-based compositional semantics for relative clauses with pied-piping is

\footnotetext{
${ }^{2}$ Partial stranding as in a boy [a picture of whom $]_{i}$ Mary made a copy of $t_{i}$ can be handled by composing a multicomponent set for whom containing a degenerate DP tree and another multi-component set for a picture of containing a degenerate DP tree. Further, the impossibility of the stranding

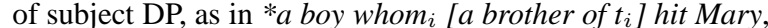
can be handled by placing an NA constraint on the subject DP dominating a trace in the relative clause tree.
} 


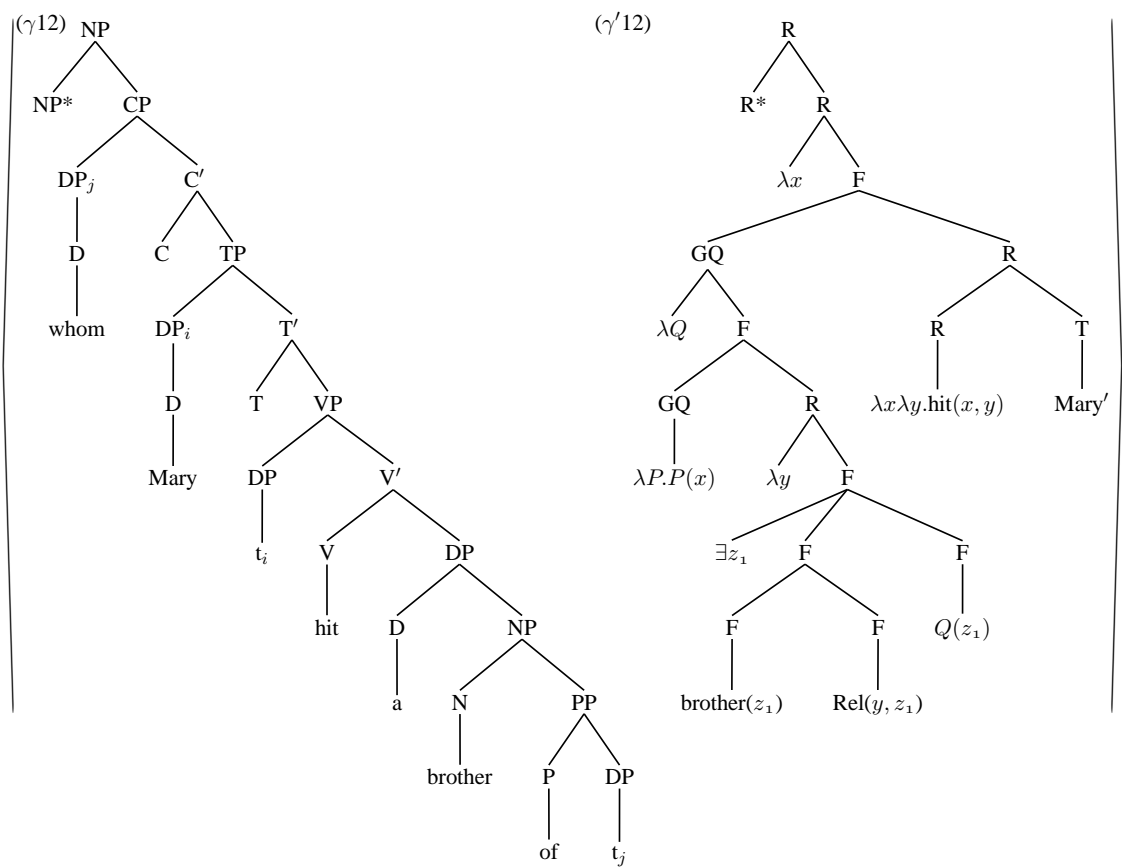

Figure 16: Derived trees for whom Mary hit a brother of

possible using examples in which the wh-word is embedded in a genitive DP, and shown that the proposed analysis can straightforwardly be extended to cases in which the wh-word is embedded in a PP. The main ingredients of the proposed analysis are: in syntax, the pied-piped material adjoins to the $w h$-word, and in semantics, the $w h$ word provides a GQ to which the meaning of the pied-piped material adjoins. I have also shown that similar analysis can handle cases in which the wh-word alone has moved to [Spec, $\mathrm{CP}$ ], stranding the rest of the DP in situ, if we use a multicomponent set containing a degenerate DP for the syntax of the relative pronoun. The proposed analysis utilizes composition operations in semantics that are already available in syntax, substitution and adjoining, thereby making syntax-semantics mapping in TAG simple and straightforward.

\section{Acknowledgment}

I thank Anoop Sarkar and the three anonymous reviewers for their insightful comments.

\section{References}

Robert Frank. 2002. Phrase Structure Composition and Syntactic Dependencies. MIT Press, Cambridge, MA.

Chung-hye Han. 2002. Compositional semantics for relative clauses in Lexicalized Tree Adjoining
Grammar. A talk presented at TAG+6, Venice, Italy, www.sfu.ca/ chunghye/papers/tag6-rc-slides.pdf.

Irene Heim and Angelika Kratzer. 1998. Semantics in Generative Grammar. Blackwell, Oxford.

Laura Kallmeyer and Tatjana Scheffler. 2004. LTAG analysis for pied-piping and stranding of whphrases. In Proceedings of TAG+7, pages 32-39, Vancouver, Canada.

Laura Kallmeyer. 2003. LTAG semantics for relative clauses. In Proceedings of the Fifth International Workshop on Computational Semantics (IWCS-5), Tilburg.

Anthony Kroch. 1989. Asymmetries in long-distance extraction in a Tree Adjoining Grammar. In Mark Baltin and Anthony Kroch, editors, Alternative Conceptions of Phrase Structure, pages 66-98. University of Chicago Press, Chicago.

Yves Schabes and Stuart M. Shieber. 1994. An alternative conception of Tree-Adjoining derivation. Computational Linguistics, pages 167-176.

Stuart Shieber and Yves Schabes. 1990. Synchronous Tree Adjoining Grammars. In Proceedings of $\mathrm{COL}$ ING'90, Helsinki, Finland.

Stuart Shieber. 1994. Restricting the weak-generative capacity of Synchronous Tree-Adjoining Grammars. Computational Intelligence, 10(4).

David Weir. 1988. Characterizing Mildly ContextSensitive Grammar Formalisms. Ph.D. thesis, University of Pennsylvania. 